\title{
Performance of Edge Windowing for OFDM under Non-linear Power Amplifier Effects
}

\author{
Çağrı Göken ${ }^{(1,2)}$ \\ ${ }^{1}$ ASELSAN Inc., Ankara, Turkey \\ ${ }^{2}$ Dept. of Electrical and Electronics Engineering \\ Bilkent University, Ankara, Turkey \\ Email: cgoken@aselsan.com.tr
}

\author{
Onur Dizdar ${ }^{(1,2)}$ \\ ${ }^{1}$ ASELSAN Inc., Ankara, Turkey \\ ${ }^{2}$ Dept. of Electrical and Electronics Engineering \\ Bilkent University, Ankara, Turkey \\ Email: odizdar@aselsan.com.tr
}

\begin{abstract}
Edge windowing is a windowing technique for Orthogonal Frequency Division Multiplexing (OFDM) signals based on the idea of using shorter cyclic prefix (CP) and longer window lengths at the edge subcarriers while keeping the symbol length fixed. In this study, we investigate the performance of OFDM signals with edge windowing under non-linear power amplifier (PA) effects by observing out-of-band (OOB) emission characteristics, average error vector magnitude (EVM) and coded block error rate (BLER) performance. We explore whether the possible gains over conventional windowing in the presence of PA is possible. We show that the edge windowing can still provide improvements over conventional windowing in terms of $\mathrm{OOB}$ emission suppression under various PA models at the expense of increased average EVM, whereas the channel coding substantially mitigates the performance loss due to inter-symbol and intercarrier interference (ISI-ICI) effects arising as a result of shorter $\mathrm{CP}$ length at the edge subcarriers.
\end{abstract}

Index Terms-OFDM, 5G, windowing, power amplifier models.

\section{INTRODUCTION}

Orthogonal Frequency Division Multiplexing (OFDM) is a type of multi-carrier modulation which is currently being employed as the trasmission scheme for various communications systems and standards. Recently, the 3GPP standardization body has agreed that $5 \mathrm{G}$ waveform for enhanced Mobile Broadband (eMBB) and Ultra Reliable Low Latency Communication (URLLC) systems will also use CP-OFDM based waveforms for downlink and uplink [1]. OFDM has considerably strong features such as enabling high data-rate communication by using low complexity equalization techniques in frequency selective channels and some well-known drawbacks including high peak to average power ratio (PAPR) and OOB emission [2].

Filtering and windowing OFDM signals at the transmitter side are well-known low-complexity techniques to achieve spectral confinement. Edge windowing proposed in [3] is a type of windowing method, which depends on the idea of using longer window length for the subcarriers at the edge of the transmission band while keeping the symbol length same for all the subcarriers. Hence, the edge subcarriers have shorter CP length as compared to that of the inner subcarriers. Edge windowing creates a trade-off between sideband suppression and the robustness to ISI and ICI effects and can provide improved spectrum characteristics at the expense of some degradation in the average EVM performance. In [4], edge windowing is also evaluated in multiple access scenarios along with various scheduling strategies for different users experiencing different channel conditions. We explain the details of this method in Section II-B.

Even though the average EVM and OOB performances of the proposed scheme are investigated in [3], the authors have not considered any non-linear PA model in their study. In practical systems, power amplifiers change the spectral characteristics of the transmitted symbol and can effect the performance of the system, especially when they are occasionally operated in non-linear region [5]. For this reason, various PAPR reduction techniques for OFDM have been investigated extensively [6], [7]. Even though operating the PA with a certain amount of back-off can help reduce the non-linearities, it limits the output transmit power. Therefore, it is important to investigate the different windowing approaches under various PA models to assess their performance for practical scenarios.

The concerns mentioned above are also crucial for the military communication systems, where the bandwidth of the communication is limited in general, hence the spectral confinement is an important aspect to be able to support more users. Operating the PAs in the linear region to reduce OOB might not be possible in order to satisfy the transmission range requirements. Furthermore, in military ad-hoc networks there might not be any central power control mechanism to prevent near-far effect. That is, while the transmitter and the receiver communicate with each other, there might be another pair nearby on the sideband causing strong interference to the received signal. Hence, the suppression of the OOB emission has even more practical importance in the ad-hoc networks. In this study, we compared the performance of the conventional windowing and the edge windowing in terms of OOB emission characteristics, average EVM and BLER performances with non-linear polynomial and Rapp PA models [14], [15]. We showed that the edge windowing can still provide improvements over conventional windowing in terms of OOB leakage, even though the suppression gain decreases compared to the case without PA. The average EVM values increase as a result of shorter CP length at the edge subcarriers, however, by forward error correction (FEC), SNR loss due to ISI-ICI effects of the edge windowing is limited to $0.5 \mathrm{~dB}$ for 64 -QAM and 


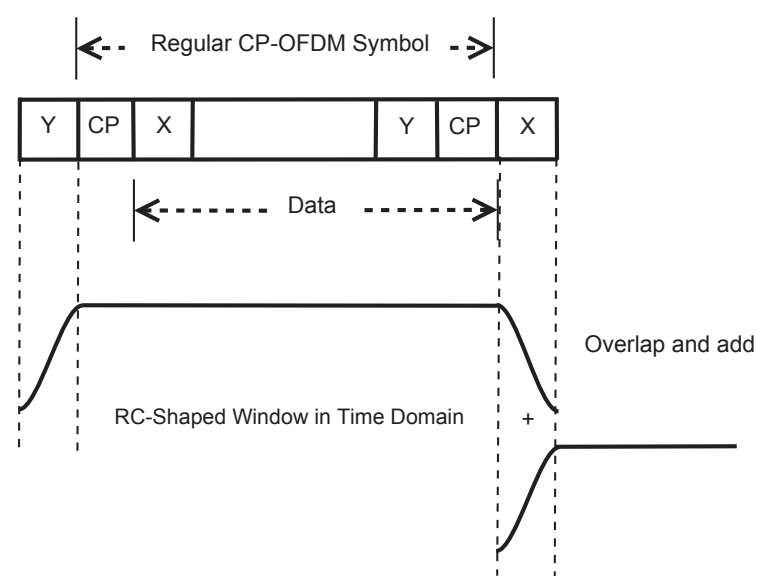

Fig. 1: Conventional windowing by using overlap and add operation over two consecutive OFDM symbols.

$0.2 \mathrm{~dB}$ for 16 -QAM modulations with a coding rate of $3 / 4$ for a target BLER level of 0.01 .

The organization of the remaining of the paper is as follows. In section II, the details of the windowing methods and the PA models used in the numerical analysis are discussed. In Section III, the simulation setting is described and the numerical results are provided. Finally, Section IV presents the concluding remarks.

\section{BACKGROUND}

\section{A. Conventional Windowing in OFDM}

Before describing the edge windowing method, we first give some details about conventional windowing approach that will be used as the reference in our comparisons. Suppose that the transmitted CP-OFDM signal uses $K$ subcarriers and let $N$, $N_{C P}, N_{W}$ denote the IFFT size, CP length and window length, respectively. In conventional windowing, the redundancy used for each subcarrier in the time domain is equal to $N_{W}+N_{C P}$ samples. Time windowing is applied by using a windowing function, such as, raised cosine shaped window [2] for an extended version of the CP-OFDM signal. The same operation is performed for each OFDM symbol and the consecutive OFDM symbols are overlapped and added over a length of $N_{W}$ samples to build the final transmission block. Fig. 1 describes the conventional windowing operation at the transmitter. $X, Y$ and $C P$ are all small portions of the data part, where they are copied and appended in the way depicted in Fig. 1. Note that the length of the CP part is $N_{C P}$ and the lengths of $X$ and $Y$ are $N_{W}$ samples each. At the receiver end, the redundant part is discarded, FFT operation is performed and the equalization is applied.

The main motivation behind using windowing as a spectral confinement technique is to prevent abrupt transitions between two consecutive OFDM symbols, as the discontinuities between the symbols in the time domain result in high sidelobe power in the frequency domain. Note that this windowing approach is also known as window overlap and add (WOLA)

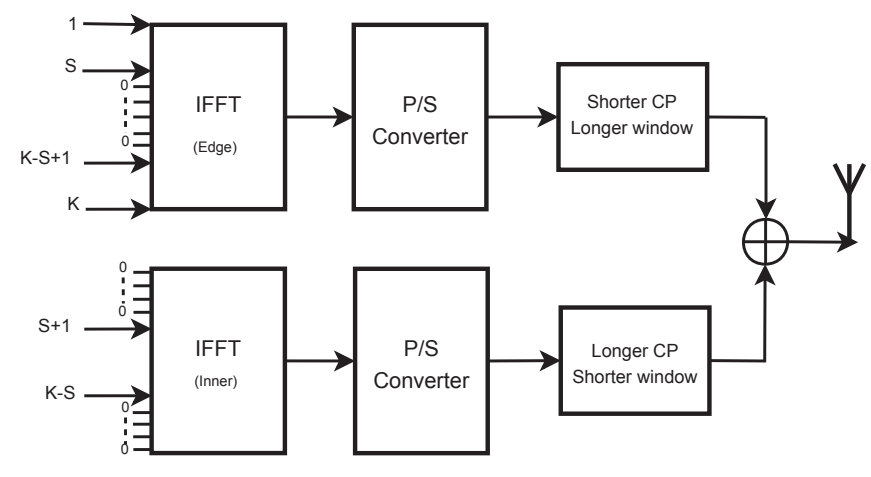

Fig. 2: Edge windowing with using different $\mathrm{CP} /$ window lengths for edge and inner subcarriers.

technique and in the remaining of the paper, it will be referred as such.

\section{B. Edge Windowing}

In edge windowing, the idea is to use different window lengths for different subcarrier groups. Basically, the subcarriers with indices $\{1,2, \ldots S, K-S+1, \ldots, K-1, K\}$ are used as the edge subcarriers and the remaining ones are used as the inner subcarriers, hence the total number of edge subcarriers is $2 S$. The length of the window and CP for the edge subcarriers are denoted as $N_{W}^{e}$ and $N_{C P}^{e}$ whereas the length of the window and CP for the inner subcarriers are denoted as $N_{W}^{i}$ and $N_{C P}^{i}$. As the overall symbol length is identical for all subcarriers, we must have $N_{W}^{e}+N_{C P}^{e}=N_{W}^{i}+N_{C P}^{i}$ with $N_{W}^{e}>N_{W}^{i}$ and $N_{C P}^{e}<N_{C P}^{i}$. Fig. 2 describes the edge windowing operation at the transmitter. At the receiver end, the operations are the same as in the case with WOLA. The total complexity of the edge windowing scheme is comparable with WOLA.

The main reason of using shorter $\mathrm{CP}$ and larger window lengths for the edge subcarriers is to achieve higher spectral suppression compared to that of WOLA while keeping the total overhead fixed, as those subcarriers have more contribution to the OOB emission. Extending the window length at the expense of shortening the $\mathrm{CP}$ length increases the vulnerability of the data carried on the edge subcarriers to ISI and ICI effects. As the number of subcarriers with shorter CP length increases, more degradation at the link level performance can be expected unless the delay spread of the channel is less than $N_{C P}^{e}$. The selection of the edge windowing parameters is important for the performance of the scheme and many possible trade-offs are discussed in [3]. In Section III, we use different $N_{W}^{e}, N_{C P}^{e}$ and $S$ values to observe the trade-offs in the presence of nonlinear PA.

\section{Non-linear Power Amplifier Models}

Non-linear PA models use analytical expressions to relate the input and output signals with the amplitude-to-amplitude modulation (AM-AM) and the amplitude-to-phase modulation (AM-PM) characteristics. Let the instantaneous input signal of 
an amplifier be defined as $x=|x| e^{j \phi_{x}}$. The signal at the output of the amplifier is written as $y=|y| e^{j\left(\phi_{x}+\Delta \phi\right)}$, where

$$
|y|=F_{\mathrm{AM}-\mathrm{AM}}(|x|), \quad \Delta \phi=F_{\mathrm{AM}-\mathrm{PM}}(|x|) .
$$

In this study, we consider two different non-linear PA models, namely polynomial and modified Rapp. The Rapp model [8] is originally proposed to represent solid-state amplifiers with an AM-AM characteristic only. A modified version of the model is given in [9] that also defines the AM-PM characteristic. We consider the simplified AM-AM and AMPM characteristics for the modified Rapp model in [15] as

$$
\begin{aligned}
& F_{\mathrm{AM}-\mathrm{AM}}(|x|)=\frac{G|x|}{\left(1+\left(\frac{G|x|}{V_{\text {sat }}}\right)^{2 p}\right)^{1 / 2 p}}, \\
& F_{\mathrm{AM}-\mathrm{PM}}(|x|)=\frac{A|x|^{q}}{\left(1+\left(\frac{|x|}{B}\right)^{q}\right)}
\end{aligned}
$$

where $G$ is the small signal gain, $p$ is the smoothness factor, $V_{\text {sat }}$ is the saturation voltage, $q$ is the smoothness factor and $A$ and $B$ are the fitting parameters. The input $|x|$ is the amplitude of the signal $x$ in Volts. The output of the AM-AM function is in Volts and the output of the AM-PM function is in degrees.

The polynomial PA model uses two separate polynomial approximations in the form

$$
F_{\mathrm{AM}-\mathrm{AM}}(z)=\sum_{i=0}^{M-1} p_{i} z^{i}, \quad F_{\mathrm{AM}-\mathrm{PM}}(z)=\sum_{i=0}^{M-1} q_{i} z^{i}
$$

for the AM-AM and AM-PM characteristics. The input $z$ is the power of the signal $x$ in $\mathrm{dBm}$. The output of the AM-AM function is also in $\mathrm{dBm}$ and the output of the AM-PM function is in degrees [14]. The polynomial coefficients $p_{i}$ and $q_{i}$ 's are specified according to the characteristics of the actual amplifier in consideration.

In our simulations, we use the parameters specified by 3 GPP in [14] and [15] for the polynomial and modified Rapp models, respectively. The parameters are given for operating frequencies below $6 \mathrm{GHz}$ in the specified documents. We consider these parameters to be realistic as they are based on measurements using real power amplifiers in mobile devices. In our simulations, we operate the amplifiers with $11.6 \mathrm{~dB}$ input back-off with respect to their $1 \mathrm{~dB}$ compression points, as suggested in [15]. The main reason of using different PA models is to test the performance of the edge windowing under different non-linearity characteristics and observe their effects to the key performance indicators.

\section{NUMERICAL RESUlts}

In this section, we present our results comparing the spectrum characteristics and error performances of the systems using WOLA and edge windowing with non-linear PAs under multipath fading channel.

\section{A. System}

The system we use in the simulations is given in Fig. 3. We use a subcarrier spacing of $15 \mathrm{kHz}$ for the OFDM signal as in
LTE. The number of subcarriers is $K=128$, corresponding to a transmission bandwidth of $1.92 \mathrm{MHz}$. The FFT size is $N=512$. We set the CP length and window length for the inner subcarriers as $N_{C P}^{i}=40$ and $N_{W}^{i}=5$ samples for the edge windowing approach. These values are also used as the $\mathrm{CP}$ and window length for WOLA. We use two different CPwindow length pairs for the edge subcarriers, that is, $N_{C P}^{e}=5$, $N_{W}^{e}=40$ or $N_{C P}^{e}=20, N_{W}^{e}=25$ to observe the performance trade-offs. We considered the cases for $S=0,20$ and 40 in our study. Note that $S=0$ corresponds to WOLA.

The channel estimator in the system is used to estimate the multiplicative channel coefficients for each OFDM subcarrier that reflects the combined effects of the multipath channel and the non-linear PA. We consider the minimum mean-squareerror (MMSE) channel estimator given in [10]. We assume the channel power delay profile (PDP) is known in the MMSE estimator in order to minimize the effects of estimation error on the presented results (We observed that despite the effects of non-linear PA on subcarriers in frequency domain, using the real channel PDP in the estimator returned better estimation performance than using a robust PDP with length equal to the delay spread of the channel). The first OFDM symbol is used as the pilot symbol to perform MMSE channel estimation. The channel is assumed to be time-invariant, so that, the estimated channel coefficients are not updated for the following OFDM symbols carrying data.

We use polar codes [11] for FEC in the system. As proved in [11], polar codes achieve channel capacity in binaryinput discrete memoryless channels (BI-DMC) with Successive Cancellation (SC) decoding. In AWGN channels, polar codes concatenated with CRC and decoded with SC-List (SCL) [12] algorithm obtain a better error performance than polar codes with SC decoding, and even 3GPP standard Turbo codes [13]. More recent studies [16]-[19] also show that polar codes have comparable performance with state-of-the-art LDPC and Turbo codes. We use a list size of $L=8$ in our simulations, since SCL-8 has negligible error performance loss with respect to larger list sizes [12] and is feasible in terms of hardware implementation aspects. We consider the $(2048,1536)$ polar code with CRC-8 in our simulations.

We performed the simulations under COST 207 Typical Urban channel with 12 taps, where the last tap of the channel occurs at $5 \mu \mathrm{s}$ [20]. Note that the CP length of 40 samples corresponds to the $\mathrm{CP}$ duration of $5.21 \mu \mathrm{s}$ for the given system parameters. The values for each channel tap are obtained from a Rayleigh distribution at every realization and the total power of the channel is normalized to 1 . We use 16-QAM and 64-QAM modulations and random interleaving before modulation. The parameters relevant to PA models are as referenced in Section II-C.

\section{B. Simulation Results}

The performance is evaluated in terms of average EVM, spectral characteristics and coded BLER.

1) Average EVM: Average EVM is calculated at the receiver side for the modulated symbols over 10000 channel realiza- 


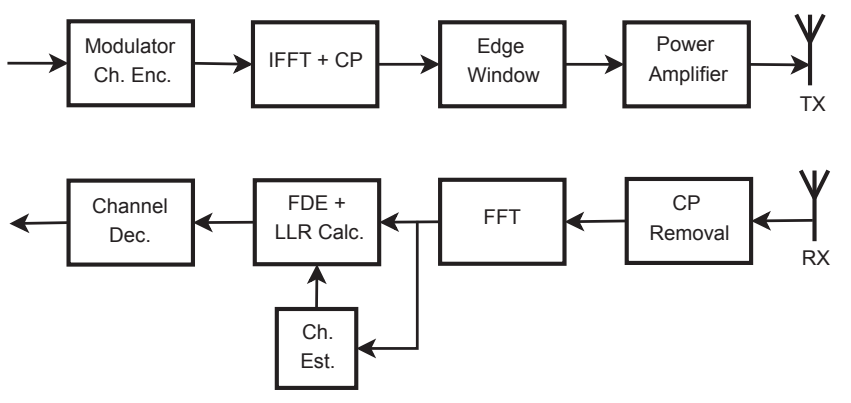

Fig. 3: System model for the simulations.

\begin{tabular}{|c|c|c|c|}
\hline$\left(N_{C P}^{e}, N_{W}^{e}, S\right)$ & No PA & Poly. & Rapp \\
\hline$(N / A, N / A, 0)$ & $0.06 \%$ & $2.82 \%$ & $2.18 \%$ \\
\hline$(20,25,20)$ & $0.43 \%$ & $2.90 \%$ & $2.27 \%$ \\
\hline$(20,25,40)$ & $0.68 \%$ & $2.99 \%$ & $2.37 \%$ \\
\hline$(40,5,20)$ & $0.89 \%$ & $3.09 \%$ & $2.49 \%$ \\
\hline$(40,5,40)$ & $1.44 \%$ & $3.38 \%$ & $2.79 \%$ \\
\hline
\end{tabular}

TABLE I: Average EVM values without PA and with polynomial PA model and Rapp PA models for QPSK.

tions. At each realization, the EVM percentage value on a given subcarrier is the square root of the ratio of the power of the error vector to the average reference constellation power (multiplied by 100). Note that the error vector is defined as the difference between the modulation constellation point and the received symbol after the equalization at the receiver. In Fig. 4, the average EVM (\%) values are shown for each subcarrier index for different windowing settings with the polynomial PA and without any PA. The ISI-ICI effects for each subcarrier in edge windowing without PA are already investigated in [3]. Basically, both ISI and ICI are effective on the edge

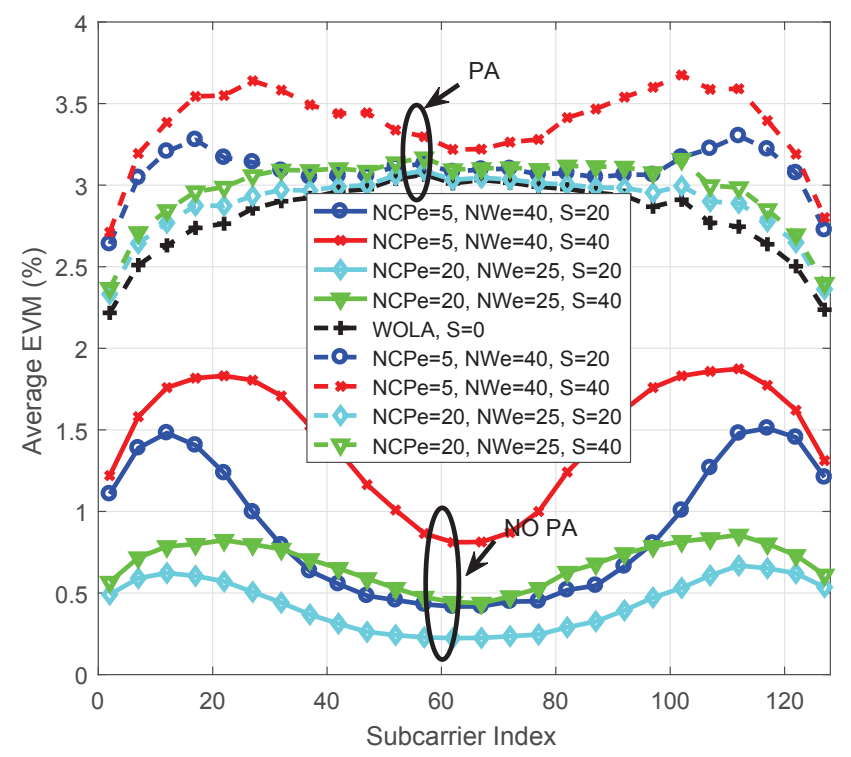

Fig. 4: Average percentage of EVM values at each subcarrier without PA and with polynomial PA. subcarriers and only ICI is effective for inner subcarriers. Hence, there is a non-uniform distribution of EVM over the subcarriers. When the polynomial PA is used, and $S=0$, the non-linear effects of the PA have a non-uniform effect over the subcarriers as well and larger EVM is observed at the inner subcarriers. When $N_{C P}^{e}=20$ and $N_{W}^{e}=25$, the PA effects are more dominant compared to the ISI-ICI effects in the edge windowing. However, for $N_{C P}^{e}=5$ and $N_{W}^{e}=40$, the resulting average EVM is similar to the case without PA in terms of EVM variations over subcarriers. This means that ISI-ICI effects resulting from using short CP length become significant.

We give the EVM values averaged over the 10000 channel realizations and all subcarriers in Table I. We also include the results obtained with the Rapp model in the table to analyze the effects of different PA models on EVM. We observe that, when there is no PA, the average EVM for WOLA is only $0.06 \%$ as the CP length is larger than the channel delay spread. It is also obvious that, using more edge subcarriers with shorter $\mathrm{CP}$ length yields higher average EVM. For example, when $S=$ $40, N_{C P}^{e}=5$ and $N_{W}^{e}=40$, average EVM becomes $1.44 \%$. The effect of the PA to the EVM values becomes significant even in the case of WOLA. However, the increase in EVM due to edge windowing is less compared to the case without PA. For instance, the setting with $S=40, N_{C P}^{e}=20$ and $N_{W}^{e}=25$ increases the EVM of WOLA by $0.17 \%$ for polynomial PA and $0.19 \%$ for Rapp model compared to $0.62 \%$ increase in the case without PA. On the other hand, the setting with $S=$ $40, N_{C P}^{e}=5$ and $N_{W}^{e}=40$ increases the EVM of WOLA by $0.56 \%$ for polynomial PA and $0.61 \%$ for Rapp model compared to $1.38 \%$ increase in the case without PA. This is due to the fact that as the non-linear effects of the PA increase, they become the dominant factor for the in-band EVM value and the ISI-ICI effects of the edge windowing become less significant.

2) $O O B$ Emission Suppression: Next, we focus on the spectral characteristics of the OFDM signal under different settings. The power spectral density (PSD) of the transmitted signal is calculated via Welch's method by using Hann window with overlap ratio of $25 \%$ and the segment length of 1012 . The results are given in Fig. 5, 6 and 7 for the cases without PA and with polynomial and Rapp PA models. ${ }^{1}$

In Fig. 5, we observe that for a fixed number of edge subcarriers, as a result of using longer window length, a rapid decrease at the beginning of the sideband is observed and the "asymptotic" performance of the windowing is achieved more quickly. On the other hand, for a given window length, increasing the number of edge subcarriers yields a better asymptotic performance. In Fig. 6 and 7, we observe the third-order product terms as an effect of non-linear PA. For both PA models, the characteristics of the different windowing settings are preserved. However, overall PSD values are better in Rapp PA model compared to the polynomial PA model. This is actually verified in EVM analysis in Section III-B1 as

${ }^{1}$ Note that normalized frequency in the figures is defined as $k / N$ where $k$ is the subcarrier index and $N=512$. Hence one subcarrier spacing corresponds to $15 \mathrm{kHz}$ and to 0.0019 in normalized frequency. 


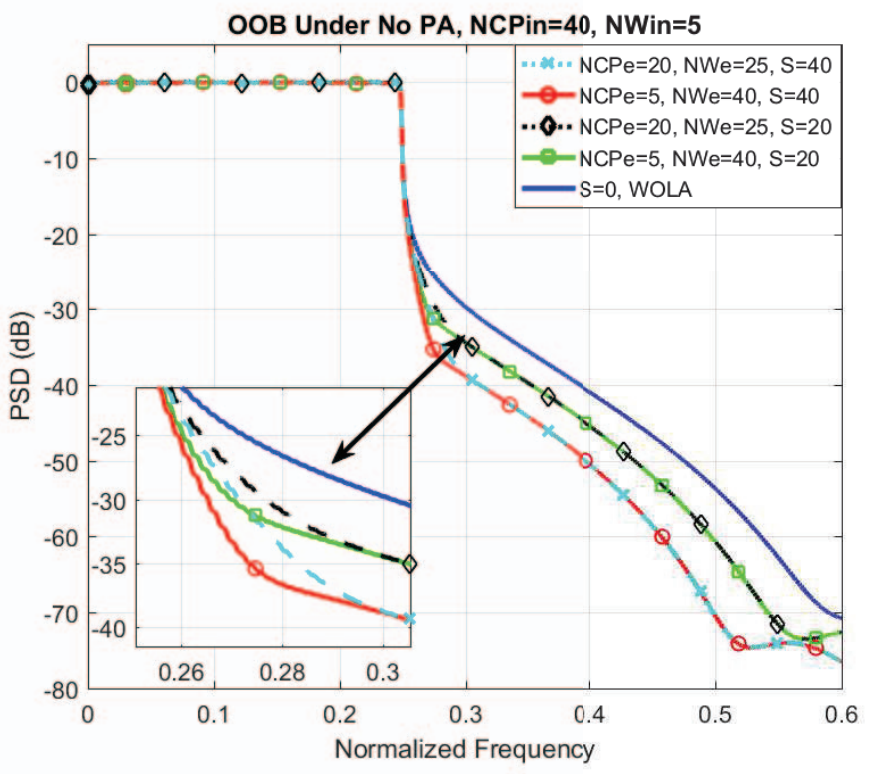

Fig. 5: PSD vs normalized frequency when there is no PA.

well, revealing that the non-linear effects are more dominant in polynomial PA model. The differences in the asymptotic performances with different edge windowing parameters are less significant in the cases with polynomial and Rapp PA compared to the case without PA. The rapid decrease at the beginning of the sideband is observed in the presence of PA as well. Hence, edge windowing is still better than WOLA in terms of OOB emission performance.

In Table II, the numerical values in terms of suppression gains over WOLA are provided for the investigated cases. It is observed that the presence of non-linear PA decreases the gain via edge windowing, however $3-6 \mathrm{~dB}$ of the suppression gain at the sideband can still be achieved for various windowing settings. Asymptotically, edge windowing brings $1.5-2.5 \mathrm{~dB}$. suppression gain for polynomial PA and $2-3.5 \mathrm{~dB}$ gain for Rapp PA model.

3) Coded BLER: Finally, we provide the coded BLER performance of different windowing approaches in Fig. 8 for 16-QAM and 64-QAM modulation with a coding rate of $3 / 4$ in the presence of polynomial PA model. We observe that for 16-QAM, the coded BLER performances of the various techniques are very close for the BLER target of 0.01 . For example, 0.01 BLER target is reached at $E_{s} / N_{0}=20.44 \mathrm{~dB}$ for WOLA case, and at $E_{s} / N_{0}=20.64 \mathrm{~dB}$ for the case of $N_{C P}^{E}=5, N_{W}^{E}=40, S=20$. Hence, the SNR loss is approximately $0.2 \mathrm{~dB}$ for this scenario. In 64-QAM, we observe that at higher SNR values, the performance difference is more observable compared to the 16-QAM case, as expected. However, the performance difference is not significant in this case as well. For example, 0.01 BLER target is reached at $E_{s} / N_{0}=25.25 \mathrm{~dB}$ for WOLA case, and at $E_{s} / N_{0}=25.75 \mathrm{~dB}$ for the case of $N_{C P}^{E}=5, N_{W}^{E}=40, S=40$. Hence, the SNR loss is at most $0.5 \mathrm{~dB}$ for this scenario. The other settings have

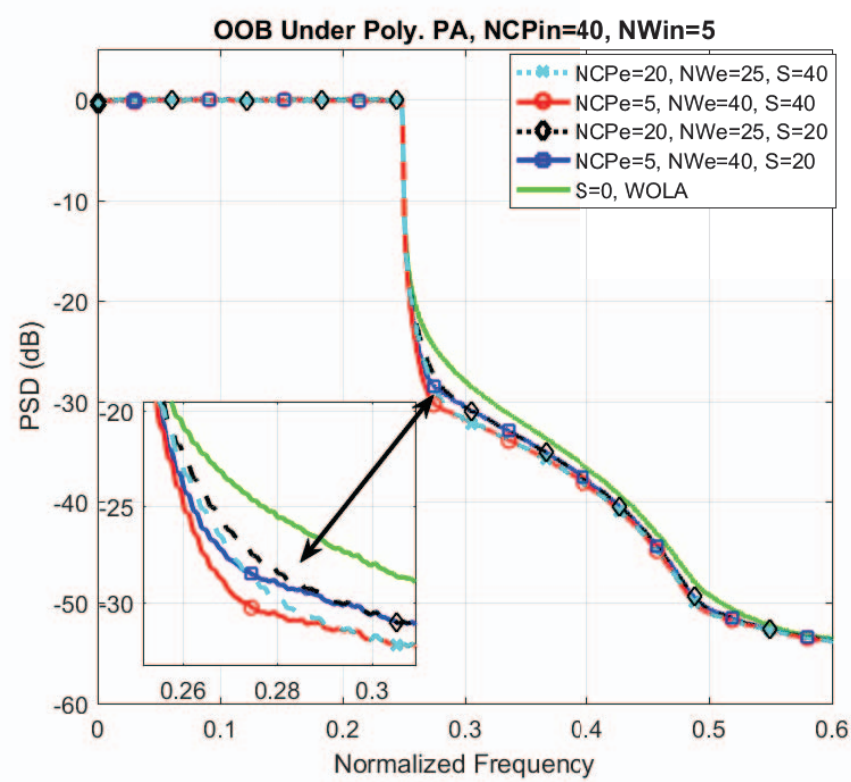

Fig. 6: PSD vs normalized frequency when there is polynomial PA.

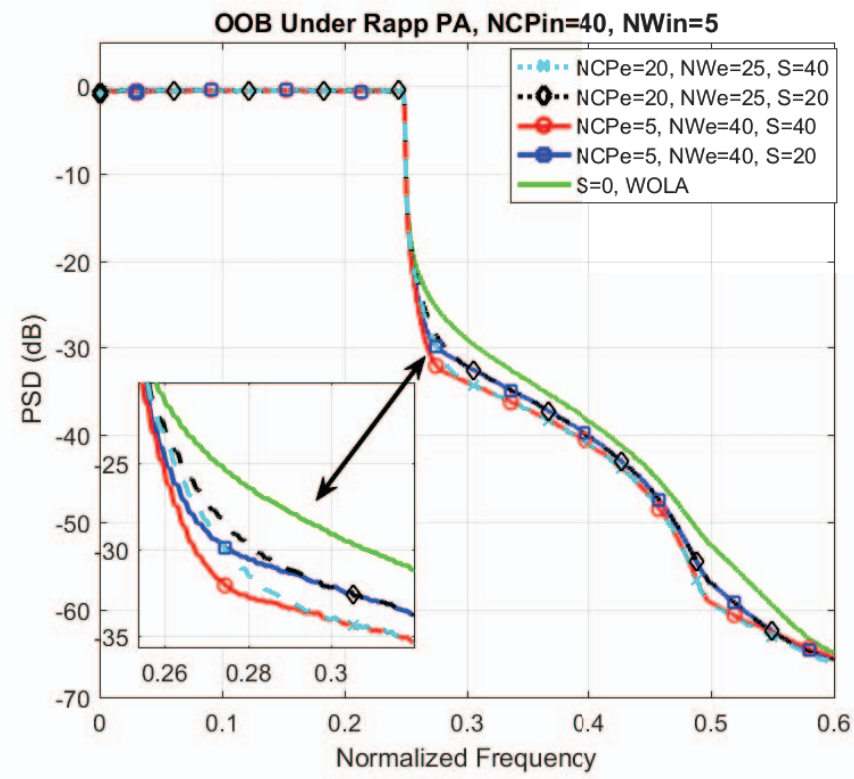

Fig. 7: PSD vs normalized frequency when there is Rapp PA.

even closer performance to the WOLA case. Especially, for $N_{C P}^{E}=20, N_{W}^{E}=25$, BLER performance is almost identical with WOLA for both 16-QAM and 64-QAM. Hence, we can conclude that even though edge windowing induces larger EVM due to the ISI-ICI effects, it is possible to compensate for such increase by using FEC with a proper coding rate. This conclusion can be verified by observing the Table I for the polynomial case. It is observed that the maximum and minimum EVM values correspond to approximately $29.4 \mathrm{~dB}$ and $31 \mathrm{~dB}$ of SIR, respectively. Such SIR values and differences are 


\begin{tabular}{|c|c|c|c|c|c|c|c|c|c|}
\hline & \multicolumn{3}{|c|}{ No PA } & \multicolumn{3}{c|}{ Polynomial PA } & \multicolumn{3}{c|}{ Rapp PA } \\
\hline$\left(N_{C P}^{E}, N_{W}^{E}, S\right)$ & $120 \mathrm{kHz}$. & $360 \mathrm{kHz}$. & $720 \mathrm{kHz}$. & $120 \mathrm{kHz}$ & $360 \mathrm{kHz}$. & $720 \mathrm{kHz}$. & $120 \mathrm{kHz}$. & $360 \mathrm{kHz}$. & $720 \mathrm{kHz}$. \\
\hline$(20,25,20)$ & $3.74 \mathrm{~dB}$ & $4.77 \mathrm{~dB}$ & $4.20 \mathrm{~dB}$ & $2.80 \mathrm{~dB}$ & $2.59 \mathrm{~dB}$ & $1.60 \mathrm{~dB}$ & $3.04 \mathrm{~dB}$ & $3.18 \mathrm{~dB}$ & $2.08 \mathrm{~dB}$ \\
\hline$(20,25,40)$ & $5.53 \mathrm{~dB}$ & $9.03 \mathrm{~dB}$ & $8.64 \mathrm{~dB}$ & $3.97 \mathrm{~dB}$ & $3.89 \mathrm{~dB}$ & $2.44 \mathrm{~dB}$ & $4.46 \mathrm{~dB}$ & $4.92 \mathrm{~dB}$ & $3.40 \mathrm{~dB}$ \\
\hline$(40,5,20)$ & $5.81 \mathrm{~dB}$ & $4.84 \mathrm{~dB}$ & $4.20 \mathrm{~dB}$ & $4.03 \mathrm{~dB}$ & $2.64 \mathrm{~dB}$ & $1.62 \mathrm{~dB}$ & $4.51 \mathrm{~dB}$ & $3.22 \mathrm{~dB}$ & $2.08 \mathrm{~dB}$ \\
\hline$(40,5,40)$ & $9.79 \mathrm{~dB}$ & $9.25 \mathrm{~dB}$ & $8.66 \mathrm{~dB}$ & $5.68 \mathrm{~dB}$ & $4.00 \mathrm{~dB}$ & $2.47 \mathrm{~dB}$ & $6.66 \mathrm{~dB}$ & $5.03 \mathrm{~dB}$ & $3.40 \mathrm{~dB}$ \\
\hline
\end{tabular}

TABLE II: Suppression resulting in PSD as compared to WOLA in the cases of Polynomial PA model, Rapp PA model and no PA for various frequency values away from edge of the in-band.

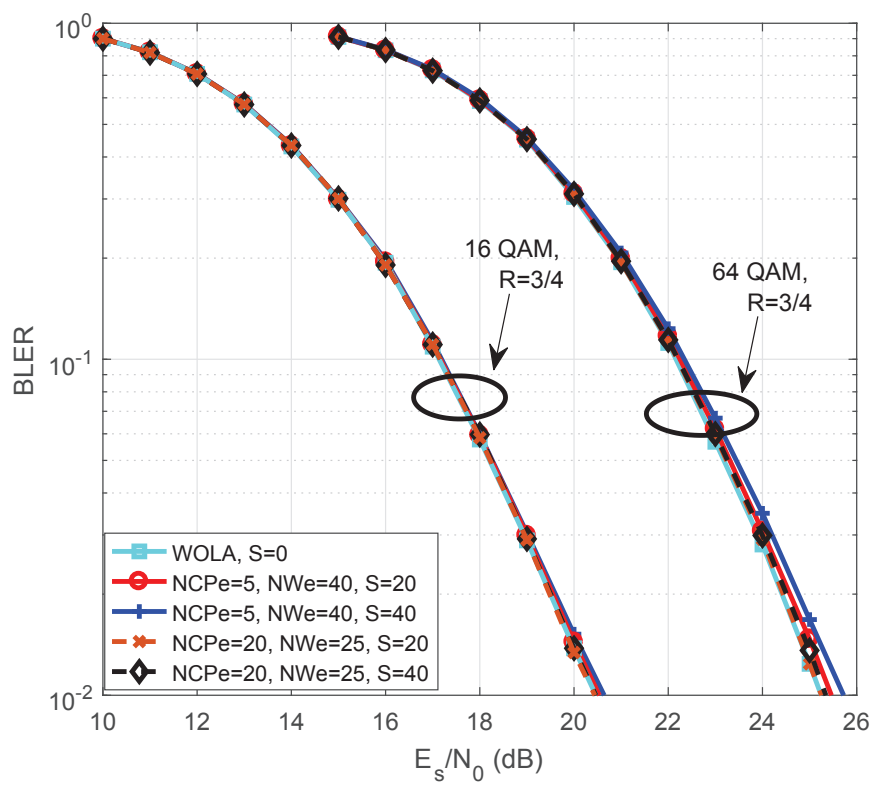

Fig. 8: BLER vs $E_{s} / N_{0}$ when there is polynomial PA for 16-QAM and 64-QAM with coding rate $3 / 4$.

negligible for the selected modulation, coding rate and BLER target according to Fig. 8. Even though the BLER analysis is performed with polynomial PA, similar results can be obtained with Rapp PA model, which are omitted due to lack of space.

\section{Conclusion}

In this study, we compared the performances of conventional windowing (WOLA) and edge windowing in the presence of the non-linear PA models. In order to evaluate the performance of the different windowing approaches, we performed the simulations by using different PA models, and provided performance results for average EVM, OOB emission, and coded BLER. We observed that, edge windowing can provide spectral suppression gains over WOLA even with the presence of a non-linear PA. The drawback of the method is the ISI-ICI effects as a result of shorter CP length at the edge subcarriers. According to selected parameters of the edge windowing, either PA non-linearities or ISI-ICI effects of the edge windowing might be more dominant for the average EVM. Finally, we showed that even if the average EVM increases, FEC can compensate for the ISI-ICI effects of the edge windowing to the BLER performance. Hence, we conclude that, edge windowing might be desirable to achieve further suppression over WOLA without losing significant link level performance for the OFDM based communication systems.

\section{REFERENCES}

[1] 3GPP, "TSG RAN, TR38.802 v1.0.0 on Study on New Radio (NR) Access Technology Physical Layer Aspects; for information“, Nov, 2016 [Available: https://portal.3gpp.org/ngppapp/CreateTdoc.aspx?mode=view\& contributionUid=RP-162202].

[2] A. Goldsmith, Wireless Communications, Cambridge Univ. Press, 2005,

[3] A. Sahin and H. Arslan, "Edge windowing for OFDM based systems," IEEE Communications Letters, vol. 15, no. 11, pp. 1208-1211, November 2011.

[4] A. Sahin and H. Arslan, "The impact of scheduling on edge windowing," 2011 IEEE Global Telecommunications Conference - GLOBECOM 2011, Houston, TX, USA, 2011, pp. 1-5.

[5] E. Costa, M. Midrio and S. Pupolin, "Impact of amplifier nonlinearities on OFDM transmission system performance," IEEE Communications Letters, vol. 3, no. 2, pp. 37-39, February 1999.

[6] R. van Nee and A. de Wild, "Reducing the peak-to-average power ratio of OFDM," VTC 98. 48th IEEE Vehicular Technology Conference,, Ottawa, Ont., 1998, pp. 2072-2076 vol.3.

[7] L. J. Cimini and N. R. Sollenberger,"Peak-to-average power ratio reduction of an OFDM signal using partial transmit sequences, “ IEEE Communications Letters, vol. 4, no. 3, pp. 86-88, March 2000.

[8] C. Rapp, "Effects of HPA-nonlinearity on a 4-DPSK/OFDM-signal for a digital sound broadcasting system," Proceedings of the Second European Conference on Satellite Communications, Liege, Belgium, Oct. 22-24, 1991, pp. 179-184.

[9] M. Honkanen and S. G. Haggman, "New aspects on nonlinear power amplifier modeling in radio communication system simulations," The 8th IEEE Intern. Symp. on Personal, Indoor and Mobile Radio Commun. Waves of the Year 2000. PIMRC '97., , vol. 3, pp. 844-848, Sep. 1997.

[10] Y. Li and L. J. Cimini and N. R. Sollenberger, "Robust channel estimation for OFDM systems with rapid dispersive fading channels," IEEE Trans. Communications, vol. 46, no. 7, pp. 902-915, July 1998.

[11] E. Arkkan, "Channel polarization: A method for constructing capacityachieving codes for symmetric binary-input memoryless channels," IEEE Trans. Inform. Theory, vol. 55, no. 7, pp. 3051-3073, July 2009.

[12] I. Tal and A. Vardy, "List decoding of polar codes," in IEEE Trans. Inform. Theory, vol. 61, no. 5, pp. 2213-2226, May 2015.

[13] K. Niu and K. Chen, "CRC-aided decoding of polar codes," in IEEE Commun. Lett., vol. 16, no. 10, pp. 1668-1671, Oct. 2012.

[14] 3GPP R4-163314, "Realistic power amplifier model for the New Radio evaluation," Nokia, Alcatel-Lucent Shanghai Bell.

[15] 3GPP R1-167779, "On DL Rapp PA model for waveform evaluation below $6 \mathrm{GHz}$," Nokia, Alcatel-Lucent Shanghai Bell.

[16] 3GPP R1-167215, "Channel coding schemes for mMTC scenario," Huawei, HiSilicon.

[17] 3GPP R1-167270, "Performance of eMBB channel coding candidates," Nokia, Alcatel-Lucent Shanghai Bell.

[18] 3GPP R1-167895, "Performance evaluation on channel coding candidates for NR," ZTE Corp., ZTE Microelectronics.

[19] 3GPP R1-166559, "Performance of channel coding schemes for NR," Intel Corporation.

[20] COST 207, "Digital land mobile radio communications," Office for Official Publications of the European Communities, Final Report, Luxembourg, 1989. 